\title{
Monitoring the Formation of Amyloid Oligomers Using Photoluminescence Anisotropy
}

Bo Jiang, Amir Aliyan, Nathan P. Cook, Andrea Augustine, Ghibom Bhak, Rodrigo Maldonado, Ashleigh D. Smith McWilliams, Erick M. Flores, Nicolas Mendez, Mohammad Shahnawaz, Fernando J. Godoy, Javier Montenegro, Ines MorenoGonzalez, Angel A. Martí

\section{Accepted Manuscript}

This is the Accepted Manuscript version of a Published Work that appeared in final form in Journal of the American Chemical Society, Copyright (c) 2019 American Chemical Society after peer review and technical edityng by the publisher. To access the final edited and published work see: https://doi.org/10.1021/jacs.9b06966

\section{How to cite:}

J. Am. Chem. Soc. 2019, 141, 39, 15605-15610

\section{Copyright information:}

(C) 2019 American Chemical Society 


\section{Detection of Oligomeric Amyloid Aggregates in Real-Time Using Metal Complexes}

Bo Jiang, ${ }^{\dagger}$ Amir Aliyan,${ }^{\dagger}$ Nathan P. Cook,${ }^{\dagger}$ Andrea Augustine, ${ }^{\dagger}$ Ghibom Bhak, ${ }^{\psi}$ Rodrigo Maldonado,${ }^{\dagger}$ Ashleigh D. Smith McWilliams, ${ }^{\dagger}$ Erick Flores,${ }^{\xi}$ Nicolas G. Mendez Dinamarca, ${ }^{\dagger}$ Fernando Godoy,${ }^{\xi}$ Javier Montenegro, ${ }^{\psi}$ Ines MorenoGonzalez, ${ }^{\ddagger}$ Angel A. Martî ${ }^{\uparrow}, \Delta, \S, *$

${ }^{\dagger}$ Department of Chemistry, ${ }^{\Delta}$ Bioengineering and ${ }^{\S}$ Materials Science \& Nanoengineering Rice University, Houston, Texas 77005, United States. ${ }^{\xi}$ Departamento de Química de los Materiales, Facultad de Química y Biología, Universidad de Santiago de Chile, Av. Libertador B. O’Higgins, 3363, Santiago Chile. ${ }^{\psi}$ Centro Singular de Investigación en Química Biolóxica e Materiais Moleculares (CIQUS), Departamento de Química Orgánica, Universidade de Santiago de Compostela, 15782 Santiago de Compostela, ${ }^{\star}$ Mitchell Center for Alzheimer’s disease and Related Brain Disorders, Department of Neurology, The University of Texas Health and Science Center, Houston, TX 77030.

Supporting Information Placeholder

ABSTRACT: The formation of oligomeric soluble aggregates is related to the toxicity of amyloid peptides and proteins. In this manuscript we report the use of a pyrazinephenanthroline metal complex $\left(\left[\mathrm{Ru}(\mathrm{bpy})_{2}(\mathrm{dpqp})\right]^{2+}\right)$ to track the formation of amyloid oligomers in real-time using photoluminescence anisotropy. Our assays show that amyloid- $\beta(A \beta)$ forms oligomers immediately after the assay is started reaching a steady state at ca. $48 \mathrm{~h}$. SDS-PAGE, DLS and TEM were used to monitor and confirm the formation of oligomers. The probe also showed to be effective to monitor the formation of $\alpha$-synuclein oligomers in realtime.

Amyloid proteins are a broad class of proteins that have minimal primary sequence similarity, yet all commonly form $\beta$-sheet rich unbranched fibrils. ${ }^{1}$ The transition from soluble proteins to insoluble aggregates is associated with a variety of diseases. Amyloid- $\beta(A \beta)$ is one of the most studied amyloid peptides, and has been correlated with the onset of Alzheimer's disease $(\mathrm{AD}){ }^{2} \mathrm{AD}$ is a neurodegenerative disorder that causes a variety of cognitive and physical disabilities. ${ }^{3}$ The amyloid cascade hypothesis details that the transition of the monomeric $A \beta$ peptide into soluble oligomers and insoluble aggregates in the brain is linked to the onset of $\mathrm{AD} .{ }^{4}$ Based on this, a neuropathological marker of $\mathrm{AD}$ development is the formation and extent of $A \beta$ plaques in the brains of $\mathrm{AD}$ patients. However, even though $\mathrm{A} \beta$ plaques are detected in the brains afflicted with $\mathrm{AD}$, recent discoveries have shown that indeed, the soluble oligomeric species are the most toxic forms and

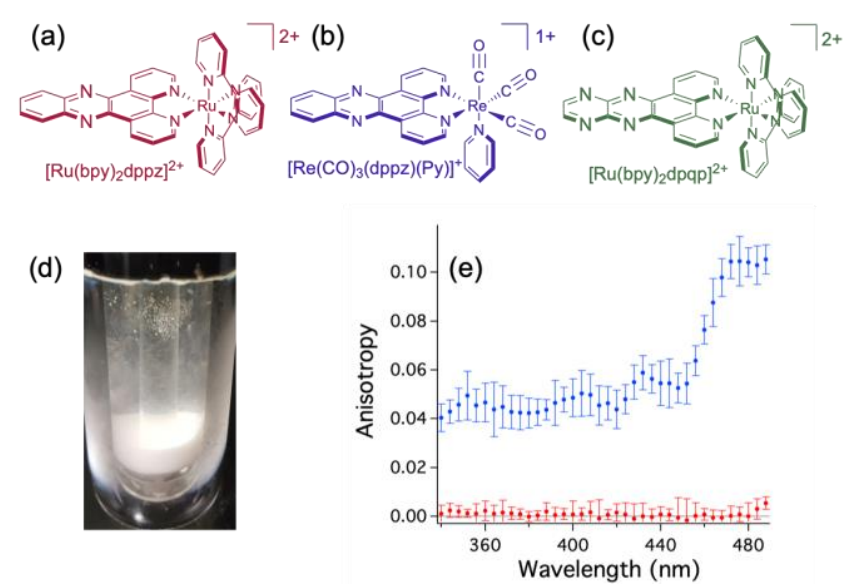

Figure 1. Structure of different metal complexes used to monitor $\mathrm{A} \beta$ aggregation (a) $\left[\mathrm{Ru}(\mathrm{bpy})_{2}(\mathrm{dppz})\right]^{2+}$ (b) $\left[\operatorname{Re}(\mathrm{CO})_{3}(\mathrm{dppz})(\mathrm{Py})\right]^{+} \quad$ (c) $\left[\operatorname{Ru}(\mathrm{bpy})_{2}(\mathrm{dpqp})\right]^{2+}$ $\left[\mathrm{Ru}(\mathrm{bpy})_{2}(\mathrm{dpqp})\right]^{2+}$ in $\mathrm{MeOH} / \mathrm{EtOH}(1: 4)$ glass at $77 \mathrm{~K}$. (e) Anisotropy of $\left[\mathrm{Ru}(\mathrm{bpy})_{2}(\mathrm{dpqp})\right]^{2+}$ at different excitation wavelengths $\left(\lambda_{\mathrm{em}}=620 \mathrm{~nm}\right)$ in glass (blue dots) and in aqueous solution at room temperature (red dots).

correlate mostly strongly with the disease progression, which gives the detection of these species marked importance. ${ }^{5,6}$

Due to the significance of monitoring the conversion of the $A \beta$ monomer to oligomer and fibrils, several fluorescence probes have been developed to study these transitions. Thioflavin T (ThT) is a widely used probe to detect $A \beta$ aggregates with a fluorescence light-switching response in the presence of $A \beta$ fibrils. ${ }^{7,8}$ However, ThT is relatively insensitive to the detection of soluble oligomers and therefore the facile detection of $A \beta$ oligomers continues to be a complicated challenge. In recent years several research groups have become 
interested in developing photoluminescent molecules capable of sensing $A \beta$ oligomers. For example, BisANS, indole dyes, and Bodipy-based probes have also been reported to detect oligomers. ${ }^{9}$ Chai and coworkers introduced an organic probe with preferential light switching response toward $A \beta$ oligomers, which was utilized for both in vitro detection of oligomers and exvivo imaging of transgenic mouse brain slices. ${ }^{10}$ In a more recent report, a multicomponent probe consisting of ThT, sulforhodamine B, and sulfo-Cy5 as well as two peptide-based recognition motifs was reported by Hatai and coworkers, and successfully used for $\mathrm{A} \beta$ oligomers detection. ${ }^{11}$ Challenges of current methodologies for the detection of oligomers in real-time include limited signal response in the presence of oligomeric aggregates, low specificity, and low binding affinity. Furthermore, most oligomer probes are based on organic dyes that have a relatively short excited-state lifetime and limited photochemical stability (compared to inorganic metal complexes). Metal complexes have unique characteristics, such as long lifetimes and high photochemical stability which lend themselves as excellent probes for biophysical assays. ${ }^{12}$

In the last few years, several reports by our group and others have shown the importance of metal dipyridophenazine complexes in the study of protein aggregation, and their pathologies. ${ }^{13}$ For example $\left[\mathrm{Ru}(\mathrm{bpy})_{2}(\mathrm{dppz})\right]^{2+}$ and $\left[\operatorname{Re}(\mathrm{CO})_{3}(\mathrm{dppz})(\mathrm{Py})\right]^{+} \quad(\mathrm{bpy}=$ 2,2'-bipyridine; $\quad \mathrm{dppz}=\operatorname{dipyrido}\left[3,2-a: 2^{\prime}, 3^{\prime}\right.$ $c$ ]phenazine, $\mathrm{Py}=$ pyridine) have shown light-switching response towards fibrillar $A \beta$ (Figure $1 \mathrm{a}-\mathrm{b}$ ).${ }^{14-17}$ In this manuscript we describe the use of the ruthenium metal complex, $\left[\mathrm{Ru}(\mathrm{bpy})_{2}(\mathrm{dpqp})\right]^{2+}\left(\mathrm{dpqp}=\right.$ pyrazino $\left[2^{\prime}, 3^{\prime}: 5,6\right]$ pyrazino[2,3-f][1,10]phenanthroline),${ }^{18}$ to detect soluble oligomeric $A \beta$ (Figure 1c). This metal complex does not present a strong light-switching response, but instead, maintains constant photoluminescence regardless of the $\mathrm{A} \beta$ aggregation state. Thus, we utilized the photoluminescence anisotropy of this complex (which is sensitive to the size of the detected molecule/macromolecule) to track the formation of large soluble oligomers of $A \beta_{1-42}$ in real-time, and introduced this technique as a powerful approach to detect amyloid oligomers.

Photoluminescence anisotropy is a technique that is proportional to the rotational correlational time of a molecule. The larger the molecule (length and volume) the slower the rotational correlation time. This effect is described by the Perrin equation which relates the measured anisotropy (r) to the rotational correlational time $(\theta):{ }^{19}$

$\frac{r_{0}}{r}=1+\frac{\tau}{\theta}$

where $r_{0}$ is the fundamental anisotropy, and $\tau$ is the molecule lifetime. The rotational correlation time can also be associated to the volume of the species under study by: ${ }^{19}$

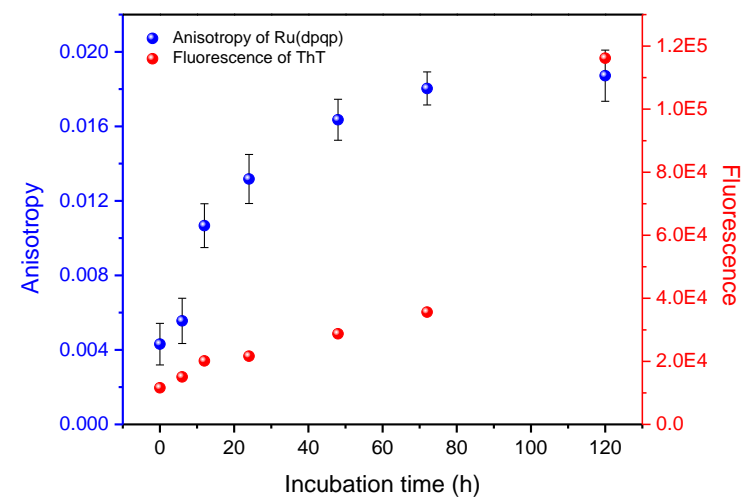

Figure 2. Real-time monitoring of $A \beta$ oligomers using photoluminescence anisotropy (blue dots) and $\mathrm{A} \beta$ fibrils using ThT fluorescence (red dots).

$\theta=\frac{\eta V}{R T}$

(eq. 2)

where $\eta$ is the viscosity, $\mathrm{V}$ the volume of the molecule, $\mathrm{R}$ the gas constant and $\mathrm{T}$ the temperature. This relationship shows that $r$ will approach to $r_{0}$ as the size of the molecule increases. In principle, a probe such as $\left[\mathrm{Ru}(\mathrm{bpy})_{2}(\mathrm{dpqp})\right]^{2+}$ will rotate very fast in solution which will result in a complete depolarization or zero anisotropy. Nonetheless, if $\left[\mathrm{Ru}(\mathrm{bpy})_{2}(\mathrm{dpqp})\right]^{2+}$ binds a large macromolecule, it will rotate at the rate of the large macromolecule, resulting in an increase in anisotropy. Importantly, the size of molecules that can be probed by fluorescence anisotropy are related to the lifetime of the probe. This is particularly important when the probe has a lifetime close in value to the rotational correlation time of the molecule (eq 1 and 2). Probes with short lifetimes (such as ThT) can only probe molecules with small rotational correlation time. However, large molecules with large rotational correlational times require probes with long lifetimes. The excited state lifetime of $\left[\mathrm{Ru}(\mathrm{bpy})_{2}(\mathrm{dpqp})\right]^{2+}$ in buffer solution is $416.7 \mathrm{~ns}$, which allows probing large soluble $A \beta$ oligomers of tenths of units.

We first determined the fundamental anisotropy for $\left[\mathrm{Ru}(\mathrm{bpy})_{2}(\mathrm{dpqp})\right]^{2+}$ in a frozen matrix $(\mathrm{MeOH} / \mathrm{EtOH}$ 1:4) in liquid nitrogen where $r_{0}=0.11$ with $480 \mathrm{~nm}$ excitation (Figure 1e). This is the maximum value of anisotropy that $\left[\mathrm{Ru}(\mathrm{bpy})_{2}(\mathrm{dpqp})\right]^{2+}$ can achieve when it is completely immobilized, and is related to the depolarization arising from the angle between the absorption and emission transition dipole moments $(\beta)$ :

$r_{0}=\frac{2}{5}\left(\frac{3 \cos ^{2} \beta-1}{2}\right)$

The calculated $\beta$ for $\left[\mathrm{Ru}(\mathrm{bpy})_{2}(\mathrm{dpqp})\right]^{2+}$ is $44^{\circ}$ for 480 $\mathrm{nm}$ excitation. The value of anisotropy as a probe changes from one environment to another with different anisotropy values, and is dictated by:

$r=\sum_{i} f_{i} r_{i}$ (eq. 4)

where $r_{i}$ are the anisotropies of the individual species (e.g. free in solution vs bound to oligomers) and $f_{i}$ are the fractional intensities. We expected 


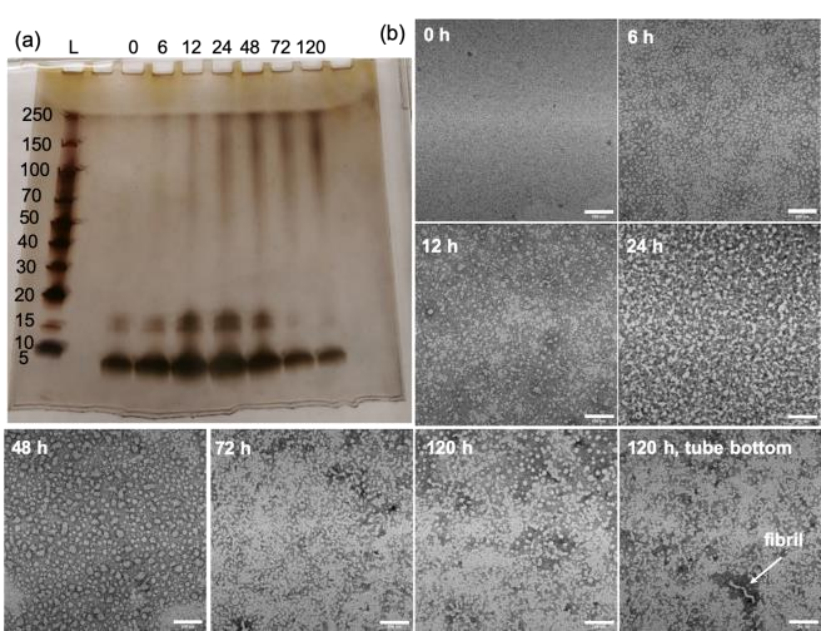

Figure 3. (a) SDS of A $\beta$ incubated for different times. The numbers on top represent the time in hours and on the left are the ladder in $\mathrm{kDa}$. (b) TEM images of aliquots taken at different time points during $A \beta$ incubation. Scale bar $=100$

$\left[\mathrm{Ru}(\mathrm{bpy})_{2}(\mathrm{dpqp})\right]^{2+}$ to present minimum anisotropy values (close to zero) when free in solution or even when interacting with monomeric $\mathrm{A} \beta$ given its relatively long lifetime in solution. Alternatively, $\left[\mathrm{Ru}(\mathrm{bpy})_{2}(\mathrm{dpqp})\right]^{2+}$ bound to oligomers was expected to present an increase in anisotropy dictated by the amount of $\left[\mathrm{Ru}(\mathrm{bpy})_{2}(\mathrm{dpqp})\right]^{2+}$ bound to oligomers and the size of the oligomers (see eqs 1-4).

The real-time detection of oligomers using the photoluminescence anisotropy of $\left[\mathrm{Ru}(\mathrm{bpy})_{2}(\mathrm{dpqp})\right]^{2+}$ is presented in Figure 2. A $\beta$ oligomers were obtained by incubating a solution of monomeric $\mathrm{A} \beta(100 \mu \mathrm{M})$ at room temperature under quiescent conditions. At different time points, aliquots were taken from the mixture and centrifuged at $1844 \mathrm{~g}$. The top part of the centrifuged was mixed with $\left[\mathrm{Ru}(\mathrm{bpy})_{2}(\mathrm{dpqp})\right]^{2+}$ to a final concentration of $1 \mu \mathrm{M}\left[\mathrm{Ru}(\mathrm{bpy})_{2}(\mathrm{dpqp})\right]^{2+}$ and 25 $\mu \mathrm{M} \mathrm{A} \beta$, while the bottom part was mixed with ThT. For $\left[\mathrm{Ru}(\mathrm{bpy})_{2}(\mathrm{dpqp})\right]^{2+}$, a rapid increase in anisotropy is observed, which starts right at the beginning of the experiment (Figure 2, blue dots). This is in contrast with the well-known sigmoidal behavior observed for the formation of $\mathrm{A} \beta$ fibrils and consistent with the data from the bottom part of the centrifuged samples (Figure 2, red dots). ${ }^{8}$ Small oligomers are known to form instantaneously, ${ }^{20}$ and are likely the reason of non-zero anisotropy signal at the beginning of the experiment. Control experiments of $\left[\mathrm{Ru}(\mathrm{bpy})_{2}(\mathrm{dpqp})\right]^{2+}$ in water (no $A \beta$ ) show anisotropy values close to zero.

We confirmed the formation of $A \beta$ oligomers using SDS-PAGE (Figure 3a). Silver stained gels show the formation of a diffuse band (resistant to SDS) that is around $170 \mathrm{kDa}$ consistent with the formation of oligomers. Lower bands corresponding to $A \beta$ monomers, as well as dimers and small oligomers due to association with SDS are also seen. The diffuse band at $170 \mathrm{kDa}$ corresponds to a distribution of oligomers of different sizes, and it is consistent with previous oligomers reports. ${ }^{21}$ These are large oligomers composed of ca. $38 \mathrm{~A} \beta_{1-42}$ monomers on average (dividing $170 \mathrm{kDa}$ by the mass of $\mathrm{A} \beta_{1-42}$ ). Qualitatively, it seems that the distribution increases in size with incubation time. Also, the oligomer band seems to get darker until reaching $48 \mathrm{~h}$ consistent with the anisotropy experiments. To explore this, Western blot experiments on glutaraldehyde cross-linked $\mathrm{A} \beta$ were performed and showed the diffuse band $170 \mathrm{kDa}$ (Figure S1). Crosslinked $\mathrm{A} \beta$ oligomers heated at $70{ }^{\circ} \mathrm{C}$ can be seen, while non-crosslinked $\mathrm{A} \beta$ did not show high order oligomers. This is a signature of the unstable nature of oligomers in general.

The formation of oligomers was also probed by using dynamic light scattering (DLS). DLS experiments (SI, Figure S2), show the assembly of $A \beta$ monomers into structures of hydrodynamic radii of ca. $20 \mathrm{~nm}$ in diameter within the first 10 hours, eventually reaching a constant size of ca. $35 \mathrm{~nm}$ after 20 hours. These results are consistent with the diffuse band with an approximate weigh of ca. $170 \mathrm{kDa}$ observed for SDS-PAGE and Western blot studies. TEM images were also obtained to characterize the shape of the formed structures (Figure $3 b)$. The images indicate small spherical aggregates at early times, which transition into larger structures at longer times. Fibrillar structures can be seen occasionally in lower part of the tube after centrifugation at $120 \mathrm{~h}$ (ca. $100 \mathrm{~nm}$ ) and with a curved appearance. These fibrillar structures have been seen before together with oligomers ${ }^{20}$ and differ from the straight fibrils obtained by the typical method of incubation at $700 \mathrm{rpm}$ and $37^{\circ} \mathrm{C}$ (Figure S3).

In order to explore the dependence of anisotropy as $\left[\mathrm{Ru}(\mathrm{bpy})_{2} \text { (dpqp) }\right]^{2+}$ binds to $\mathrm{A} \beta_{1-42}$ oligomers, a titration experiment was performed (Figure 4). Here the anisotropy of $A \beta_{1-42}$ oligomers (48 hours incubation) with different concentrations of $\left[\mathrm{Ru}(\mathrm{bpy})_{2}(\mathrm{dpqp})\right]^{2+}$ was obtained. The results can be fitted to a one-site model solving the mass balance law (eq. 5):

$$
\begin{aligned}
& r=r_{R u-f}+\frac{r_{b}-r_{f}}{2[R u]}\left(\left(k_{d}+[R u]+\frac{[A \beta]-[A \beta]_{m}}{n}\right)-\right. \\
& \left.\sqrt{\left(k_{d}+[R u]+\frac{[A \beta]-[A \beta]_{m}}{n}\right)^{2}-4 \frac{[A \beta]-[A \beta]_{m}}{n}[R u]}\right)
\end{aligned}
$$

where $r_{f}$ and $r_{b}$ are the anisotropy of free and bound $\left[\mathrm{Ru}(\mathrm{bpy})_{2}(\mathrm{dpqp})\right]^{2+}$ respectively, $[R u]$ is the concentration of $\left[\mathrm{Ru}(\mathrm{bpy})_{2}(\mathrm{dpqp})\right]^{2+},[A \beta]$ is the total concentration of $\mathrm{A} \beta$, which is $50 \mu \mathrm{M},[A \beta]_{m}$ is the concentration of monomeric $A \beta, n$ is the number of $A \beta$ units in an oligomer (set as 37 based in SDS-PAGE studies) and $k_{d}$ is the dissociation constant in $\mu \mathrm{M}$. The value of $r_{b}$ was chosen to be 0.09 based on our DLS results and the projected value of anisotropy for oligomers using equation 1 and 2. Based on this model, the $\left[R u(b p y)_{2}(d p q p)\right]^{2+}$ dissociation constant is ca. 34 $\mathrm{nM}$. 


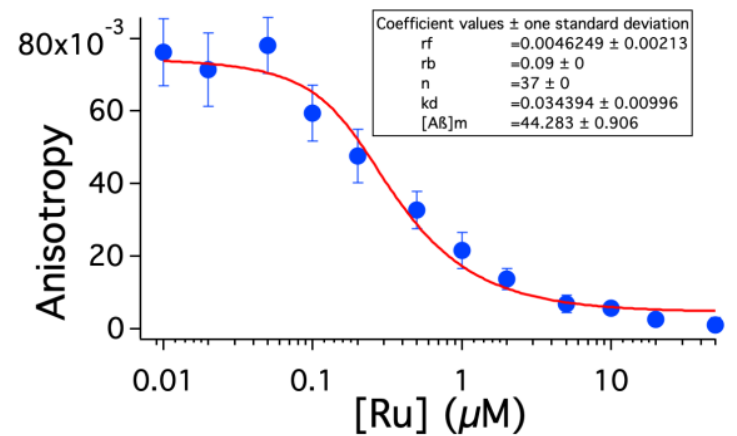

Figure 4. Anisotropy of different concentrations of $\left[\mathrm{Ru}(\mathrm{bpy})_{2}(\mathrm{dpqp})\right]^{2+}$ with constant $\mathrm{A} \beta$ concentration (50 $\mu \mathrm{M})$. The red line is a fit to equation 5 .

Given the fact that the importance of oligomer detection is due to their toxicity, it was highly relevant to check whether the detected species were indeed toxic. Cell viability assay was performed using N2a cell line treated with different $A \beta$ forms: monomers, oligomers formed after 24 and 48 hours, and fibrils. As expected, cells treated with $\mathrm{A} \beta$ oligomers showed minimal viability $(\sim 60 \%)$, while those treated with fibrils and monomers exhibited higher viability ( $\sim 80 \%$, Figure S4). The toxicity of monomers can be attributed to their ability to form oligomers during the treatment process, while that of fibrils may be explained by the likelihood of the fibril solution to contain oligomeric species. Nevertheless, the findings strongly suggest that the detected species are toxic, and the further emphasizes the ability of this probe in Alzheimer's disease research.

Importantly, the size of molecules that can be probed by fluorescence anisotropy are related to the lifetime of the probe. ${ }^{22}$ The $\alpha$-synuclein $(\alpha S)$ oligomers, associated with the onset of Parkinson's disease, have been shown to be meta-stable species lacking $\beta$-sheet conformation and that cannot be detected with the classical ThT probe. $^{23,24}$ However, early detection of $\alpha \mathrm{S}$ oligomeric precursors is of critical importance due to their high fibrillation propensity and pathological potential. ${ }^{22,23}$ Therefore, to test the scope of $\left[\mathrm{Ru}(\mathrm{bpy})_{2}(\mathrm{dpqp})\right]^{2+}$ for the detection of oligomeric aggregates of different proteins, we performed aggregation assays with $\alpha \mathrm{S}$. Real-time aggregation assays with both $\left[\mathrm{Ru}(\mathrm{bpy})_{2}(\mathrm{dpqp})\right]^{2+}$ anisotropy and ThT fluorescence show sigmoidal behaviors for both assays (Figure S5), however $\left[\mathrm{Ru}(\mathrm{bpy})_{2}(\mathrm{dpqp})\right]^{2+}$ anisotropy starts detecting the formation of aggregates about 20 hours earlier than ThT, which is consistent with the production of $\alpha \mathrm{S}$ oligomers.

In summary, our studies indicate that $\left[\mathrm{Ru}(\mathrm{bpy})_{2}(\mathrm{dpqp})\right]^{2+}$ binds to large soluble oligomers, leading to an increase in rotational correlational time, and therefore an increase in anisotropy. The formation of oligomers was confirmed using SDS-PAGE, Western Blot, TEM, and DLS techniques. Monitoring the anisotropy of $\left[\mathrm{Ru}(\mathrm{bpy})_{2}(\mathrm{dpqp})\right]^{2+}$ allows us to track the formation of $A \beta$ oligomers in real-time, using simple and well known spectroscopy tools. In the future, probes can be designed having different photoluminescent lifetimes that will allow the detection of oligomers of different sizes. Real-time monitoring of oligomers can provide mechanistic information on their formation and kinetics. Furthermore, a quick and reliable methodology for detecting oligomers is of importance in identifying small molecules capable of inhibiting or delaying the formation of oligomers, with important consequences for the treatment of amyloid diseases.

\section{ASSOCIATED CONTENT}

Supporting Information. Materials and Methods and supporting spectral information. The Supporting Information is available free of charge on the ACS Publications website.

\section{AUTHOR INFORMATION}

\section{Corresponding Author}

*amarti@rice.edu

\section{ACKNOWLEDGMENT}

We thank the Welch Foundation (C-1743) for financial support.

\section{REFERENCES}

(1) Greenwald, J.; Riek, R. Structure 2010, 18, 1244-60.

(2) Ross, C. A.; Poirier, M. A. Nat. Med. 2004, 10 Suppl, S10-7.

(3) Alzheimer's \& Dementia 2019, 15, 321-387.

(4) Karran, E.; Mercken, M.; De Strooper, B. Nat. Rev. Drug Discov. 2011, 10, 698-712.

(5) Walsh, D. M.; Selkoe, D. J. J. Neurochem. 2007, 101, 1172-1184.

(6) Glabe, C. G. Neurobiol. Aging 2006, 27, 570-575.

(7) LeVine, H., 3rd Protein Sci. 1993, 2, 404-10.

(8) Cook, N. P.; Marti, A. A. ACS Chem. Neurosci. 2012, 3, 896-9.

(9) Reinke, A. A.; Seh, H. Y.; Gestwicki, J. E. Bioorg. Med. Chem. Lett. 2009, 19, 4952-7.

(10) Teoh, C. L.; Su, D.; Sahu, S.; Yun, S. W.; Drummond, E.; Prelli, F.; Lim, S.; Cho, S.; Ham, S.; Wisniewski, T.; Chang, Y. T. J. Am. Chem. Soc. 2015, 137, 13503-9.

(11) Hatai, J.; Motiei, L.; Margulies, D. J. Am. Chem. Soc. 2017, 139, 2136-2139.

(12) Martí, A. A. J. Photochem. Photobiol. A: Chem. 2015, 307-308, 3547.

(13) Chen, K.; Cui, M. Medchemcomm 2017, 8, 1393-1407.

(14) Cook, N. P.; Torres, V.; Jain, D.; Martí, A. A. J. Am. Chem. Soc. 2011, 133, 11121-11123.

(15) Cook, N. P.; Ozbil, M.; Katsampes, C.; Prabhakar, R.; Marti, A. A. J. Am. Chem. Soc. 2013, 135, 10810-6.

(16) Aliyan, A.; Kirby, B.; Pennington, C.; Marti, A. A. J. Am. Chem. Soc. 2016, 138, 8686-9.

(17) Aliyan, A.; Paul, T. J.; Pennington, C.; Sharma, G.; Prabhakar, R.; Martí, A. A. Chem 2017, 3, 898-912.

(18) Sun, Y.; Collins, S. N.; Joyce, L. E.; Turro, C. Inorg. Chem. 2010, 49, 4257-62.

(19) Lakowicz, J. R. Principles of Fluorescence Spectroscopy; Springer: New York, 2006, 741-755.

(20) Stine, W. B., Jr.; Dahlgren, K. N.; Krafft, G. A.; LaDu, M. J. J. Biol. Chem. 2003, 278, 11612-22. 
(21) Salvadores, N.; Shahnawaz, M.; Scarpini, E.; Tagliavini, F.; Soto C. Cell Rep 2014, 7, 261-8.

(22) Liu, Y.; Wolstenholme, C. H.; Carter, G. C.; Liu, H.; Hu, H.; Grainger, L. S.; Miao, K.; Fares, M.; Hoelzel, C. A.; Yennawar, H. P.; Ning, G.; Du, M.; Bai, L.; Li, X.; Zhang, X. J. Am. Chem. Soc. 2018, 140, 7381-7384.
(23) Cremades, N.; Cohen, S. I.; Deas, E.; Abramov, A. Y.; Chen, A. Y.; Orte, A.; Sandal, M.; Clarke, R. W.; Dunne, P.; Aprile, F. A.; Bertoncini, C. W.; Wood, N. W.; Knowles, T. P.; Dobson, C. M.; Klenerman, D. Cell 2012, 149, 1048-59.

(24) Bhak, G.; Lee, S.; Kim, T. H.; Lee, J. H.; Yang, J. E.; Joo, K.; Lee, J.; Char, K.; Paik, S. R. Sci Rep 2018, 8, 14295. 